\title{
Sensations Reported During Urodynamic Bladder Filling in Spinal Cord Injury Patients Give Additional Important Information
}

\author{
Jean-Jacques Wyndaele ${ }^{1}$, Michel Wyndaele ${ }^{2}$, Peter F.W.M. Rosier ${ }^{2}$ \\ ${ }^{1}$ Faculty GGW, University of Antwerp, Brasschaat, Belgium \\ ${ }^{2}$ Department of Urology, University Medical Center Utrecht, Utrecht, The Netherlands
}

Purpose: This study investigated the sensations reported during filling cystometry in patients with spinal cord lesions (SCLs) of different levels and completeness.

Methods: In this retrospective cohort study, information was gathered on patients' age and sex, cause of SCL, American Spinal Injury Association Impairment Scale (AIS), and lower urinary tract-related sensations in daily life. Filling cystometry (videourodynamics) was performed following the International Continence Society Good Urodynamic Practice Guidelines. In addition to bladder filling sensations (first sensation of bladder filling, first desire to void, strong desire to void), other sensations, such as detrusor overactivity related sensation and pain, were noted.

Results: In total, 170 patients were included (age, $45 \pm 17$ years; 114 males and 56 females, 92 with complete and 78 with incomplete SCL). The test was done $6 \pm 4$ years post-SCL. Sensation was reported by $57 \%$ of all patients. Half of the patients with complete SCL (46 of 92) had sensation, while 36\% of those with incomplete SCL (28 of 78) reported no sensation. Bladder awareness was not predictable by the AIS. The filling sensations reported were equivalent to those given in the terminology of ICS. Pain was seldom present (6\%, 10 of 170), and detrusor overactivity contraction was felt by 45 of 78 (58\%). Very few patients used sensory information for bladder management at home.

Conclusions: After SCL, most patients retained the ability to be aware of the lower urinary tract, and were assessable and gradable during urodynamic testing. The filling sensations were not different from those described in healthy individuals, but the number and sequence of the sensations were altered in a minority of patients. Pain and a sensation of unstable contractions gave additional important information. As different sensations relate to different spinal afferent pathways, the sensory evaluation during cystometry provided additional important information on the spinal cord's condition.

Keywords: Bladder sensation; Filling cystometry; Spinal cord lesion; Sensory pathways

- Research Ethics: This study was approved by Institutional Review Board (IRB) of Antwerp University Hospital (IRB No. 001176).

- Conflict of Interest: No potential conflict of interest relevant to this article was reported.

\section{INTRODUCTION}

The lower urinary tract (LUT) has extensive peripheral innervation that transfers afferent information from its different parts to the spinal cord and brain [1]. The International Continence Society (ICS) and the International Urodynamic Basic Spinal Cord Injury Data Set have standardized terminology on sensations of bladder filling [2-4]: the first sensation of bladder
Corresponding author: Jean-Jacques Wyndaele (iD https://orcid.org/0000-0002-0879-6854 Faculty GGW, University Antwerp, Bredabaan 322930 Brasschaat Belgium Email: Wyndaelejj@skynet.be

Submitted: December 22, 2020 / Accepted after revision: February 11, 2021
This is an Open Access article distributed under the terms of the Creative Commons Attribution Non-Commercial License (http://creativecommons.org/licenses/by-nc/4.0/) which permits unrestricted non-commercial use, distribution, and reproduction in any medium, provided the original work is properly cited. 
filling (FSF) is the feeling when the individual first becomes aware of bladder filling; first desire to void (FDV) is the first feeling that the individual may want to pass urine at the next convenient moment, but this can be delayed if necessary; and strong desire to void (SDV) is the persistent desire to void without the fear of leakage.

Other sensations during bladder filling include pain (an intense uncomfortable sensation in the lower abdomen/bladder) and the sensation of contractions during detrusor overactivity (DOA) (e.g., urgency or a rapid sensation of pressure). Each of these sensations is triggered by a different mechanism (stretch vs. nociceptive stimulation vs. pressure), originating from different sites in the LUT, conducted through different types of nerve fibers (A-delta or C-fibers), different peripheral nerves, and different spinal cord pathways [5].

After a spinal cord lesion (SCL), LUT dysfunction will develop in approximately $90 \%$ of patients [6], and the pathophysiology depends on the lesion's level and completeness. Bladder proprioception is likely to be lost in traumatic and nontraumatic SCL affecting the dorsal tracts. However, clinical observations have indicated that the bladder filling sensation may depend on afferent potentials transported via other routes than those involved in classical detrusor stretch proprioception. Non-bladder-related awareness, as occurs with autonomic dysreflexia (AD), was not investigated in this paper.

LUT dysfunction is among the most dangerous hazards in SCL patients, causing a high rate of readmissions and a substantial negative impact on the prognosis and the quality of life [7]. To fully understand the impact of SCL on LUT function, information on the remaining sensory function is essential. We evaluated the bladder sensations reported during filling cystometry in a cohort of SCL patients. Our study aimed to explore sensory types and prevalence, as well as to study the clinical value of bladder filling-related sensory information in patients with SCL

\section{MATERIALS AND METHODS}

We present a retrospective cohort analysis of patients with chronic SCL who underwent video-urodynamics (VUD) during 2 consecutive years.

Patients' age and sex, time since SCL, the cause and level of SCL, the American Spinal Injury Association Impairment Scale (AIS) score, the method of bladder emptying, and LUT-related sensations in daily life were collected from the records. Previous diseases of the urinary tract and drug intake were noted. The exclusion criteria were fever, signs/symptoms suggestive of urinary tract infection (UTI), hematuria, inflammation in the pelvic region, refractory $\mathrm{AD}$, and insufficient patient cooperation and cognitive function as evaluated by the clinician [8].

VUD was performed following the Good Urodynamic Practice Guidelines of ICS [9], either as part of routine follow-up or prompted by a change in symptoms. Five-channel urodynamic equipment was used (Medical Measurement Systems, Gladbeck, Germany). Filling cystometry was done through a transurethral $8 \mathrm{~F}$ double-lumen catheter connected to an external transducer. A radiocontrast solution (Urografin 30\%; Schering AG, Berlin, Germany) at room temperature was used to fill the bladder at a continuous flow rate of $30 \mathrm{~mL} / \mathrm{min}$ (infusion pump) and with the filling bag hanging from a load cell [9]. Patients were asked to report and describe all sensations between the start and end of bladder filling. The filling was stopped at a maximum capacity of $550 \mathrm{~mL}$ (volume of the infusion bottle, excluding diuresis) if no or little bladder pressure developed. The filling was stopped earlier if neurogenic DOA (NDOA) occurred with leakage, if detrusor pressure consistently rose above $40 \mathrm{~cm} \mathrm{H}_{2} \mathrm{O}$, if the patient reported a strong desire to void, or if pain occurred.

The sensations of bladder filling were categorized as FSF, FDV, SDV [4], and pain or DOA sensation (DOAS). Patterns were classified as the absence of a sensation, the presence of 1 type of filling sensation, or any combination of 2 or more sensations. An investigator was present during the entire cystometry process, making it possible to ask additional questions to define further or specify the reported sensations if needed.

After the urodynamic test, cystoscopy was done in all patients, as per our standard of care, and renal ultrasonography was performed in 114 patients. One sachet of fosfomycin $(3 \mathrm{~g})$ was given after the test to prevent UTI.

The statistical analysis was carried out with IBM SPSS Statistics ver. 27.0 (IBM Co., Armonk, NY, USA), using the chi-square test for categorical variables and 1-way analysis of variance (ANOVA) for continuous variables. Statistical significance was set at $\mathrm{P}<0.05$.

\section{RESULTS}

Fifteen patients met the exclusion criteria, which left $170 \mathrm{pa}-$ tients to be included (age, $45 \pm 17$ years, 114 males and 56 females). The time since SCL was $6 \pm 4$ years. The indication for 
Table 1. Cause of spinal cord lesion

\begin{tabular}{lc}
\hline Cause of spinal cord lesion & No. of patients \\
\hline Road traffic accident & 73 \\
Fall & 34 \\
Sports & 14 \\
Infection & 11 \\
Vascular & 6 \\
Spine surgery & 15 \\
Myelopathy & 4 \\
Tumor & 5 \\
Work accident & 6 \\
Disc herniation & 1 \\
Gun shot & 1 \\
Total & 170 \\
\hline
\end{tabular}

filling cystometry was routine follow-up in 109 patients and an evaluation due to changes in urological and other conditions in 55 patients. The indication was missing for 6 patients. The distributions of cause, level, and completeness of SCL, as well as the method of bladder emptying, are provided in Tables 1-3.

Table 4 presents an overview of bladder sensations during filling cystometry in patients with different levels and completeness of SCL, the types of sensations reported, and the eventual combinations. The numbers of filling sensations are given as 1 to 3 , but these numbers do not necessarily correspond to FSF, FDV, and SDV, as explained below. No sensation was reported by $43.5 \%$ (74 of 170 ) of patients, while $33.5 \%$ (57 of 170 ) reported more than 1 sensation. Fifty percent (46 of 92) of patients with complete (AIS A) SCL and 36\% (28 of 78) of those with incomplete (AIS B-D) SCL reported no sensation. The sensation patterns were not related to maximum cystometric capacity $(\mathrm{P}=0.115)$ or to bladder compliance from the start to the end of filling except DOA contractions $(\mathrm{P}=0.406)$.

When only 1 sensation of bladder filling was reported $(n=36-$ with or without pain or DOA) this was described as FSF by 31 patients (86\%), FDV by 3 patients, and SDV by 2 patients. When 2 sensations of bladder filling were reported $(n=22-$ with or without pain or DOA), this was described as FSF+FDV by 21 patients and FSF+SDV by 1 patient.

No difference was found between groups with 1,2, or 3 bladder filling sensations (without pain or DOA) for volume and detrusor pressure at FSF, FDV, and SDV (Table 5). Both volume and detrusor pressure increased significantly between the consecutive sensations (ANOVA $\mathrm{P}<0.001$ ) and were not signifi-
Table 2. Level and completeness of SCL

\begin{tabular}{lcc}
\hline AIS score completeness & Spinal cord level & Number \\
\hline AIS A (complete) & C1-C8 & 31 \\
& T1-T9 & 39 \\
& T10-L1 & 16 \\
& L2-S3 & 3 \\
AIS B-D (incomplete) & Cauda & 3 \\
& C1-C8 & 38 \\
& T1-T9 & 14 \\
& T10-L1 & 13 \\
Total & L2-S3 & 9 \\
\hline
\end{tabular}

AIS, American Spinal Injury Association Impairment Scale.

Table 3. Method of bladder emptying

\begin{tabular}{lc}
\hline Method & No. of patients \\
\hline Clean intermittent catheterization & 68 \\
Indwelling transurethral catheter & 54 \\
Suprapubic catheter & 15 \\
Suprapubic tapping & 40 \\
Crede/Valsalva & 3 \\
Normal voiding & 19 \\
Complete incontinence & 1 \\
Total & 170 \\
\hline
\end{tabular}

cantly different among patients with different levels and completeness of SCL (P-values between 0.105 and 0.992) (detailed data not shown).

DOA was present in 78 patients, and contractions were felt by 45 (58\%). The mean volume and pressure at which the DOAS was reported were $188 \pm 132 \mathrm{~mL}$ and $55 \pm 38 \mathrm{~cm} \mathrm{H}_{2} \mathrm{O}$, respectively. A filling sensation was present in 54 of 78 patients with DOA and in 34 of 71 patients without DOA (chi-square $\mathrm{P}=0.006$ ). A filling sensation was more likely to be reported in the absence of DOAS (chi-square $\mathrm{P}=0.008$ ).

Pain during cystometry was reported by 10 patients (6\%). Subsequent urethrocystoscopy revealed pathological findings in 5 cases: severe inflammation from the indwelling catheter in 1 case, bladder stones in 3 cases, and a polyp behind the trigone in 1 case. In the other 5 cases, cystoscopy indicated no abnormalities. Pathological findings on cystoscopy were also present in $40.5 \%$ (30 of 74) of patients with a complete absence of sen- 
Table 4. Overview of bladder sensations during filling cystometry in different level and completeness of spinal cord lesion and eventual combinations

\begin{tabular}{|c|c|c|c|c|c|c|c|c|c|c|c|}
\hline \multirow{2}{*}{ Bladder sensation } & \multicolumn{5}{|c|}{ AIS A } & \multicolumn{5}{|c|}{ AIS B-D } & \multirow{2}{*}{ Total } \\
\hline & $\mathrm{C} 1-\mathrm{C} 8$ & T1-T9 & T10-L1 & L2-S3 & Cauda & $\mathrm{C} 1-\mathrm{C} 8$ & T1-T9 & T10-L1 & L2-S3 & Cauda & \\
\hline Absent & 18 & 19 & 6 & 2 & 1 & 13 & 3 & 7 & 3 & 2 & 74 \\
\hline Pain only & 0 & 1 & 0 & 0 & 0 & 1 & 0 & 1 & 1 & 0 & 4 \\
\hline 1 Sensation of bladder filling & 2 & 6 & 5 & 1 & 0 & 6 & 4 & 1 & 1 & 0 & 26 \\
\hline 2 Sensations of bladder filling & 2 & 3 & 0 & 0 & 1 & 5 & 2 & 0 & 0 & 0 & 13 \\
\hline 3 Sensations of bladder filling & 1 & 2 & 1 & 0 & 1 & 3 & 2 & 2 & 1 & 0 & 13 \\
\hline DOAS & 3 & 2 & 0 & 0 & 0 & 4 & 0 & 0 & 0 & 0 & 9 \\
\hline 1 Sensation of bladder filling + DOAS & 1 & 1 & 2 & 0 & 0 & 1 & 1 & 1 & 0 & 0 & 7 \\
\hline 2 Sensations of bladder filling + DOAS & 2 & 0 & 0 & 0 & 0 & 3 & 0 & 1 & 1 & 0 & 7 \\
\hline 3 Sensations of bladder filling + DOAS & 1 & 3 & 2 & 0 & 0 & 3 & 1 & 0 & 1 & 0 & 11 \\
\hline 1 Sensation of bladder filling + pain & 0 & 1 & 0 & 0 & 0 & 0 & 0 & 0 & 0 & 2 & 3 \\
\hline 2 Sensations of bladder filling + pain & 1 & 0 & 0 & 0 & 0 & 0 & 0 & 0 & 1 & 0 & 2 \\
\hline 3 Sensations of bladder filling + pain & 0 & 1 & 0 & 0 & 0 & 0 & 0 & 0 & 0 & 0 & 1 \\
\hline Total & 31 & 39 & 16 & 3 & 3 & 39 & 13 & 13 & 9 & 4 & 170 \\
\hline
\end{tabular}

DOAS, sensation of detrusor overactive contraction; The numbers of sensations of filling is given as 1 to 3, not necessarily corresponding with the First sensation of filling, First desire to void, Strong desire to void sequence. AIS=American Spinal Injury Association Impairment Scale (AIS), A represents a complete lesion, B-D different types of sensory and/or motor incomplete lesions. Chi-square $\mathrm{p}=0.045$.

Table 5. Volume and pressure at the different sensations of bladder fullness in patients

\begin{tabular}{|c|c|c|c|c|c|}
\hline \multirow{2}{*}{ Volume \& pressure } & \multicolumn{4}{|c|}{ Sensations of bladder fullness during cystometric bladder filling } & \multirow{2}{*}{ P-value (ANOVA) } \\
\hline & 1 Sensation & 2 Sensations & 3 Sensations & Mean & \\
\hline FSF volume (mL) & $214 \pm 108$ & $204 \pm 90$ & $221 \pm 136$ & $214 \pm 113$ & 0.871 \\
\hline FSF pressure $\left(\mathrm{cm} \mathrm{H}_{2} \mathrm{O}\right)$ & $25 \pm 26$ & $26 \pm 19$ & $20 \pm 18$ & $23 \pm 21$ & 0.584 \\
\hline FDV volume $(\mathrm{mL})$ & $323 \pm 45$ & $283 \pm 116$ & $265 \pm 121$ & $276 \pm 115$ & 0.670 \\
\hline FDV pressure $\left(\mathrm{cm} \mathrm{H}_{2} \mathrm{O}\right)$ & - & $32 \pm 22$ & $24 \pm 17$ & $28 \pm 19$ & 1.185 \\
\hline SDV volume $(\mathrm{mL})$ & $310 \pm 199$ & 250 & $325 \pm 129$ & $313.2 \pm 133$ & 0.334 \\
\hline $\mathrm{SDV}$ pressure $\left(\mathrm{cm} \mathrm{H}_{2} \mathrm{O}\right)$ & - & - & - & $33 \pm 26$ & NA \\
\hline P-value & & & & $<0.001$ & \\
\hline
\end{tabular}

Values are presented as mean \pm standard deviation.

ANOVA, analysis of variance; FSF, first sensation of bladder filling; FDV, first desire to void; SDV, strong desire to void; NA, not available.

The number of sensations reported are given and the type of sensation to which this number corresponded.

sation during bladder filling, and in 39.5\% (38 of 96) of patients with sensation during bladder filling but without pain $(\mathrm{P}=0.625)$.

Five patients reported a sensation of bladder fullness in daily life, 2 reported pain, and 5 reported urgency. A urodynamic filling sensation was present, respectively, in 2, 1 , and 2 patients. Five of these 12 patients used this sensory information to guide bladder emptying in daily life, 2 for clean intermittent catheterization, and 3 for normal voiding, corresponding to only $3 \%$ of the study group.
Consecutive filling sensations in patients with a cauda and conus lesion were perceived at volumes of $294 \pm 49.5,346 \pm 9.2$, and $362 \mathrm{~mL}$, and at pressures of $52 \pm 7.7,21 \pm 14$, and $32 \mathrm{~cm} \mathrm{H}_{2} \mathrm{O}$, respectively. These were not significantly different from the volume and pressure values recorded for patients with SCL at other levels $(\mathrm{P}=0.309-0.988)$.

Renal ultrasonography showed changes in 19 of 114 patients (minor pyelocaliceal dilatation in 11, hydronephrosis in 3, renal cysts in 2, renal atrophy in 2, and signs of previous pyelonephri- 
tis in 1), of whom 7 had a filling sensation and 12 did not $(\mathrm{P}=$ $0.263)$.

\section{DISCUSSION}

The sensory innervation of the LUT is complex and involves autonomic and somatosensory peripheral nerves running to the lower parts of the spinal cord and ascending to the brainstem and the cortex. There is increasing knowledge of the bladder wall mechanisms involved in capturing local changes as stretches of the wall, pressure, nociceptive impulses, and the different nervous pathways used to transfer this information upwards [10-12]. However, the mechanisms through which interacting receptors and pathways create sensations during bladder filling remain to be fully elucidated [13]. It is relevant to recognize that the bladder afferent system has diverse pathways, and that it is plausible to distinguish between afferent activity and sensory information and to include a central interpretation when reported sensations are explored [14]. Bladder emptying based on LUT sensations is a process under cognitive control, influenced by multiple elements, of which bladder awareness is one [15].

SCL induces neurogenic bladder dysfunction in most patients and poses a significant threat if the sensation of a full bladder or overactive detrusor contractions remains unnoticed while high detrusor pressures develop.

SCL causes important changes in afferent innervation, beyond the lesion itself during and after the spinal shock phase: plasticity in bladder C-fiber afferent nerves have been shown in animals and humans [16]. The mechanisms generating awareness may be intensified or lost after SCL.

The spinal cord pathways for LUT sensory impulses have been well described, but it remains uncertain which peripheral nerves conduct each specific filling sensation $[5,17]$. Considering the topography of different nerves, it seems likely that a specific filling sensation may be carried by more than 1 type of peripheral nerve. Knowledge of spinal cord pathways is more extensive: the fasciculi gracili conduct signals caused by stretching (bladder filling), the anterior spinothalamic tracts transmit afferent stimuli from contractions, and the lateral spinothalamic tracts conduct noxious stimuli as pain [18]. An SCL may entirely or partially destroy 1 or more sensory pathways. Evaluating the sensations reported during cystometry can help determine which spinal pathways are most probably still active/intact and which are not. This needs further confirmation, but could potentially aid in a more detailed/accurate classification of SCL patients.

In our cohort of patients with different levels and completeness of SCL, most patients reported bladder sensations during filling cystometry. Remarkably, half of those with a complete SCL (AIS A) reported at least 1 bladder sensation, confirming that the somatomotor typing (AIS score) underevaluates the sensation of the LUT perceived by the patient [19]. The types of filling sensations and their sequence were mostly the same as given in the terminology reports. It has been documented that the different sensations of bladder filling are recognized by patients with SCL without great difficulty, as they represent what was familiar before the lesion. They are reproducible and are communicated to the investigator without difficulty [20]. In the present cohort, roughly a quarter (25 of 96) of patients with preserved sensation reported all 3 (standard) consecutive sensations of bladder filling, while the others showed different combinations of 1 or 2 sensations. Such different patterns of sensation after SCL have been described before [20]. Our findings agree with those of previous studies suggesting that after an SCL, qualitative and quantitative changes occur in bladder sensations compared to what had been felt before the lesion [21]. We found no significant difference between volumes or detrusor pressure in different patterns of sensation of bladder filling. Our data also do not confirm that the sensations of patients with complete cauda and conus lesions are only perceivable at a very high volume threshold.

A previous study in a cohort of patients with nontraumatic spinal cord myelopathy showed that $46 \%$ voided guided by sensations of bladder filling [22]. Another study with ambulatory urodynamics in patients with SCL described that $73 \%$ reported bladder sensation daily, and $41 \%$ experienced DOA [23]. Only a minority of patients reported LUT sensations in daily life in our study, and few used this information at home to guide their bladder emptying (7\%, 12 of 170$)$. A reason may be that no attention was given to these sensations during rehabilitation.

Reitz [21] found that in patients with a complete cauda or lower conus lesion, sensory input from the bladder was preserved, while all sensation was absent in patients with AIS A lesions below T6 and above T12. Furthermore, Ersoz and Akyuz [24] found that sensation was preserved in twice as many patients with a complete lesion under T10 compared with patients with a complete lesion below T11. These findings imply that in such cases, sensation during bladder filling is probably transferred through the intact hypogastric plexus to the thoracolum- 
bar spinal cord. In our study, $48 \%$ of patients with a complete lesion above the thoracic-lumbar level reported sensation, compared to $58 \%$ with a complete lesion under this level. This finding shows that in AIS-complete SCL above the inflow level of the hypogastric plexus, sensory nervous pathways may continue to function [25].

Ersoz and Akyuz [24] found clinical value in using sensations to guide bladder emptying, and Ersöz and Sayılır [26] found that preserving the sensation of bladder filling had a preventive effect on upper urinary tract deterioration in chronic SCL patients. In our cohort, pathology of the upper urinary tract was found on renal ultrasonography in $11 \%$ of patients, with no statistically significant difference between those with and without sensation during bladder filling. Therefore, more factors should probably be considered, such as the method of bladder emptying, UTI, reflux, intravesical pressure development, and drug intake.

Data on emptying and incontinence volumes and/or medication use were not included in the present study due to its retrospective nature and its specific scope and aim.

SCL can produce NDOA due to reorganization from a spinobulbospinal loop to a predominantly spinal network [27]. It was shown that DOA could be felt by half of nonneurogenic patients [28]. In our cohort, $42 \%$ of SCL patients with DOA felt a contraction. Pathological patterns of bladder filling sensation were found more frequently in those who had no DOAS, confirming that more severe spinal cord pathology exists when DOA contractions are not felt [28].

Pain during cystometry does not often occur in patients with SCL (6\% in our population), but most clinicians would consider pain as a warning sign to investigate further possibilities, such as UTI (excluded in this study), stones, catheter-induced irritation, and other conditions. In our study, pathological outcomes of cystoscopy were noted in $44 \%$ and $41 \%$ of patients with an absence of pain and absence of overall sensation, respectively, indicating that pathology in the LUT often does not induce pain during cystometry in SCL patients.

The role of bladder sensations in the urological management of patients with SCL is currently minimal, both in diagnosis and in treatment. Increasing the use of bladder sensations in diagnosis and using urodynamics as biofeedback for patients in relation to their usual emptying routine (if intermittent) would be easy and worthwhile, as this sensory information conveys a substantial amount of information related to LUT innervation, bladder activity, and possible complications.
The most frequently used technique to empty the bladder, intermittent catheterization, is mostly performed on a schedule, although systems have been explored to better link bladder fullness and time to empty [29]. If bladder filling sensations were used, this would theoretically permit more than half of the patients to schedule catheterizations when a physiological need is present. In patients with continuous leakage or an indwelling (transurethral or suprapubic) catheter, sensation plays no apparent role in bladder emptying management.

Regarding limitations of this study, many of the patients in our cohort used antimuscarinic, spasmolytic, or analgetic drugs, and some previously had botulinum toxin injections in the bladder. The 1-time measurement made in the present study did not permit an analysis of the influence of these factors on filling sensations in the short and long term. Furthermore, we had no detailed information on the brain function, but the investigator subjectively assessed cognitive problems and lack of patient compliance, and the presence of these issues served as a contraindication for participation in this study. Another limitation of this study is that it is a retrospective study based on medical records. However, there were few missing data, and all available data were obtained from standardized urodynamic tests with identical questions about sensations in a high-volume setting. As stated before for urodynamics in general, more research is also needed on the sensory component of function [30]. Examples of urodynamic curves with sensation patterns can be found in the textbook Urodynamic Testing after SCI: A Practical Guide [8].

In conclusion, as the spinal cord pathways responsible for different LUT sensations are grossly known, sensations reported during cystometry may indicate the presence or absence of these pathways. There is a need for further research on the preservation, reoccurrence, and neurological rerouting of LUT sensations in combination with an in-depth analysis of the type of sensations and awareness reported after SCL, as well as the possible applications and implications for diagnosis and bladder management. Our data confirm that some sensation or awareness from the LUT after SCL is preserved or reoccurs more often than previously reported. The findings of the present study also show that the filling sensations described in the terminology reports are present after SCL. Pain and DOAS provide additional information about spinal cord pathways. 


\section{AUTHOR CONTRIBUTION STATEMENT}

- Conceptualization: JW

- Data curation: JW

- Formal analysis: $J W$

- Methodology: JW

- Project administration: JW

-Writing-original draft: $J W$

- Writing-review \& editing: JW, MW, PFWMR

\section{ORCID}

Jean-Jacques Wyndaele

Michel Wyndaele

Peter F.W.M. Rosier

0000-0002-0879-6854

0000-0003-1757-2569

0000-0003-0445-4563

\section{REFERENCES}

1. Birder L, Wyndaele JJ. From urothelial signalling to experiencing a sensation related to the urinary bladder. Acta Physiol (Oxf) 2013; 207:34-9.

2. Biering-Sørensen F, Craggs M, Kennelly M, Schick E, Wyndaele JJ. International urodynamic basic spinal cord injury data set. Spinal Cord 2008;46:513-6.

3. Pannek J, Kennelly M, Kessler TM, Linsenmeyer T, Wyndaele JJ, Biering-Sørensen F. International spinal cord injury urodynamic basic data set (version 2.0). Spinal Cord Ser Cases 2018;4:98.

4. Abrams P, Cardozo L, Fall M, Griffiths D, Rosier P, Ulmtyen U, et al. The standardization of terminology of lower urinary tract function: report from the Standardisation Sub-committee of the International Continence Society. Neurourol Urodyn 2002;21:167-78.

5. Jänig W, Morrison JF. Functional properties of spinal visceral afferents supplying abdominal and pelvic organs, with special emphasis on visceral nociception. Prog Brain Res 1986;67:87-114.

6. Apostolidis A, Drake MJ, Emmanuel A, Gajewski J, Hamid R, Heesakkers J, et al. Neurologic urinary and faecal incontinence. In: Abrams P, Cardozo L, Wagg A, Wein A, editors. Incontinence. 6th ed. Tokyo: ICUD ICS; 2016. p. 1218.

7. Myers JB, Lenherr SM, Stoffel JT, Elliott SP, Presson AP, Zhang C, et al. Patient reported bladder related symptoms and quality of life after spinal cord injury with different bladder management strategies. J Urol 2019;202:574-84.

8. Wyndaele JJ, Kovindha A. Urodynamic testing after spinal cord injury. A practical guide. Cham: Springer International Publishing; 2017. Chapter 3. p. 9-14.
9. Rosier PFWM, Schaefer W, Lose G, Goldman HB, Guralnick M, Eustice S, et al. International Continence Society Good Urodynamic Practices and Terms 2016: Urodynamics, uroflowmetry, cystometry, and pressure-flow study. Neurourol Urodyn 2017;36:1243-60.

10. Delhaye BP, Long KH, Bensmaia SJ. Neural basis of touch and proprioception in primate cortex. Compr Physiol 2018;8:1575-602.

11. Fry CH, Sui GP, Kanai AJ, Wu C. The function of suburothelial myofibroblasts in the bladder. Neurourol Urodyn 2007;26(6 Suppl):914-9.

12. Wiseman OJ, Fowler CJ, Landon DN. The role of the human bladder lamina propria myofibroblast. BJU Int 2003;91:89-93.

13. Janssen DAW, Schalken JA, Heesakkers JPFA. Urothelium update: how the bladder mucosa measures bladder filling. Acta Physiol (Oxf) 2017;220:201-17.

14. Gillespie JI, van Koeveringe GA, de Wachter SG, De Vente J. On the origins of the sensory output from the bladder: the concept of afferent noise. BJU Int 2009;103:1324-33.

15. Harvey J, Finney S, Stewart L, Gillispie JL. The relationship between cognition and sensation in determining when and where to void: the concept of cognitive voiding. BJU Int 2012;110:1756-61.

16. de Groat WC, Yoshimura N. Changes in afferent activity after spinal cord injury. Neurourol Urodyn 2010;29:63-76.

17. Wyndaele JJ, Wyndaele M. Combining different evaluations of sensation to assess the afferent innervation of the lower urinary tract after SCI. Spinal Cord 2021;59:201-6.

18. Kamikawa K, Kuro M. Analysis of lateral column units related to vesical reflexes. Exp Neurol 1962;6:1271-84.

19. Alexander MS, Biering-Sorenson F, Bodner D, Brackett NL, Cardenas $\mathrm{D}$, Charlifie $\mathrm{S}$, et al. International standards to document remaining autonomic function after spinal cord injury. Spinal Cord 2009;47:3643.

20. Wyndaele JJ. Investigation of afferent nerves of the lower urinary tract in patients with 'complete' and 'incomplete' spinal cord injury. Paraplegia 1991;29:490-4.

21. Reitz A. Afferent pathways arising from the lower urinary tract after complete spinal cord injury or cauda equina lesion: clinical observations with neurophysiological implications. Urol Int 2012;89:462-7.

22. New PW, Dillon L. Neurogenic bladder and urodynamic outcomes in patients with spinal cord myelopathy. Top Spinal Cord Inj Rehabil 2015;21:250-6.

23. Martens FM, van Kuppevelt HJ, Beekman JA, Rijkhoff NJ, Heesakkers JP. Limited value of bladder sensation as trigger for conditional neurostimulation in spinal cord injury patients. Neurourol Urodyn 2010;29:395-400.

24. Ersoz M, Akyuz M. Bladder-filling sensation in patients with spinal 
cord injury and the potential for sensation-dependent bladder emptying. Spinal Cord 2004;42:110-6.

25. Shin JC, Chang WH, Jung TH, Yoo JH, Park SN. The determination of sensation-dependent bladder emptying time in patients with complete SCL above T11. Spinal Cord 2008;46:210-5.

26. Ersöz M, Sayılır S. Protective effect of preserved bladder-filling sensation on upper urinary tract in patients with spinal cord injury. Neurol Sci 2014;35:1549-52.

27. Steers WD. Pathophysiology of overactive bladder and urge urinary incontinence. Rev Urol 2002;4(Suppl 4):S7-18.
28. Wyndaele JJ, van Meel TD, De Wachter S. Detrusor overactivity: does it represent a difference if patients feel the involuntary contractions? J Urol 2004;172:1915-8.

29. Lombardi G, Musco S, Celso M, Ierardi A, Nelli F, Del Corso F, et al. Intravesical electrostimulation versus sacral neuromodulation for incomplete spinal cord patients suffering from neurogenic nonobstructive urinary retention. Spinal Cord 2013;51:571-8.

30. Rosier PFWM, Kuo HC, Agro EF, Gammie A, Kakizaki H, Hashim H, et al. Urodynamic testing. In: Abrams P, Cardozo L, Wagg A, Wein A, editors. Incontinence. 6th ed. Tokyo: ICUD ICS; 2016. P. 599-670. 